\title{
Three-dimensional model of non-Newtonian fluid flow in the rectangular channel
}

\section{Eduard Biletskyi ${ }^{1}$, Olena Petrenko ${ }^{2}$, Dmytro Semeniuk ${ }^{2}$}

1 - Kharkiv institute of Trade and Economy of Kyiv National University of Trade and Economy, Kharkiv, Ukraine;

2 - Kharkiv State University of Food Technology and Trade, Kharkiv, Ukraine

\section{Keywords:}

Fluid

Non-newtonian

Couette

Flow

Rheology

Model

Article history:

Received 26.02.2016

Received in revised form 12.08 .2016

Accepted 01.09.2016

Corresponding author:

Eduard Biletskyi

E-mail:

bileckyj@meta.ua

\section{Abstract}

Introduction. The three-dimensional mathematical model of non-Newtonian fluid flow was introduced, with viscosity that depends of shear velocity in rectangular channels of food equipment.

Materials and methods. Applying the method of superposition allows to build a field of longitudinal flow of non-Newtonian fluid in rectangular channel of technological equipment with moving borders and thus to define values of velocity and pressure at any point inside the channel.

Results and discussion. We developed a model of longitudinal flow of non-Newtonian fluid in rectangular channels on borders of which different longitudinal velocities are set. The model is based on the solution of one-dimensional problem of the Couette flow in the channel. The composition of flows in the slit channels with mutually perpendicular pair of borders allows to receive a flow rate formula which satisfies the principle of limit correspondence between the currents in a rectangular and slit channels. We suggest a method of construction of the velocity field which is a subdivision of the final section of the channel into sections with a different dependence on the coordinates so that in some areas the velocity depends only on a single coordinate, while in others - only on the other coordinate. We obtain the equations of lines delimiting these areas, and how to determine the shape of boundaries.

Conclusion. The analytical formulas allow defining macrokinetic characteristics of each channel at the boundary with arbitrary distribution of velocities of the flow of non-Newtonian fluid the viscosity of which depends on the shear speed. 


\section{Introduction}

The movement of non-linear fluids is a part if hydrodynamics for laminar or Stokes flows. In technical literature there are a lot of ways to solve problems related to flow of viscoplastic fluids. The objective of any solution of the problem of fluid flow is to find the pressure and the velocity vector at each point within the channel $[1,2,3]$.

\section{Materials and methods}

Three-dimensional flows are usually studied using the numerical methods. Results obtained this way are more accurate. To generalize it in order to isolate the impact of individual parameters the analysis of large amount of numerical information is required. The form of representing results of such impacts is mostly descriptive and may contain many errors. There is another approach to the problem of constructing a picture of the three-dimensional flow, the purpose of which is the analytical solution of a problem, which is simpler yet retains all the important parameters of the flow. Usually this problem is onedimensional. Then, a three-dimensional solution to the problem is constructed using heuristics compositions based on the results of one-dimensional problem solving. Then, a three-dimensional solution to the problem is constructed using heuristics compositions based on the results of one-dimensional problem solving. This solution has a lower accuracy than the numerical one, but allow the researcher to obtain physically reasonable parameter combinations as opposed to random combinations of a descriptive nature, which are obtained by numerical solution of the problem. Solutions based on the analytical methods have more methodical value, and can be used by other researchers for a variety of other tasks. As a means of studying the movement of nonlinear fluids authors adhere to the analytical approach followed by composition [4]. The basic problem in this case is the problem of the Couette flow in a slit channel. Rectangular channel and boundary conditions that create a three-dimensional flow in it are shown on Figure 1.

Knowledge of pressure and velocity at each interior point of the channel makes it possible to calculate such quantities: flow rate, the dissipative heat, shear rate and shear stress $[5,6]$.

Speed pressure fields are totally dependent on the boundary conditions on the channel walls. These conditions are determined by the construction of the working chamber through the helix lead angle of the worm and its pitch and speed of the machine shaft $[1,7,8]$.

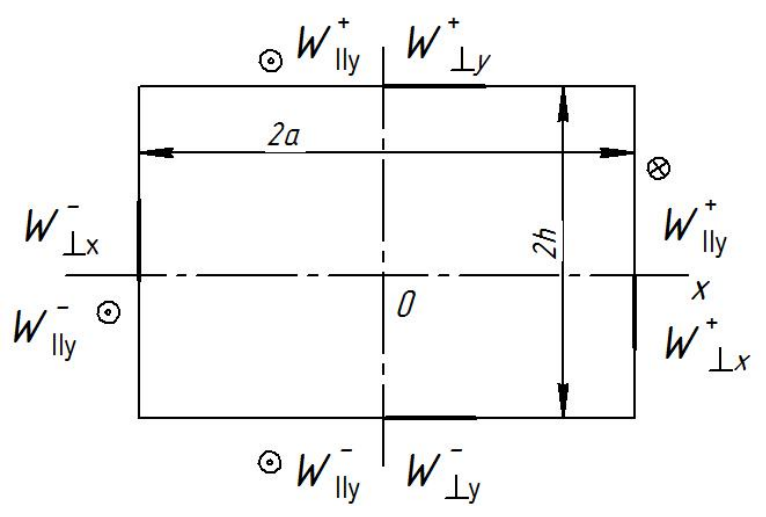

Figure 1. Rectangular channel and the boundary conditions of three-dimensional flow in channel:

$W_{I I Y}^{ \pm}$- value of longitudinal velocity on the channel borders which are perpendicular to OY axis;

$W_{I I X}^{ \pm}$- value of longitudinal velocity on the channel borders which are perpendicular to OX axis; $W_{\perp X}^{ \pm}, W_{\perp Y}^{ \pm}$- value of transverse velocities on the channel borders 
The boundary conditions for the basic problem of Couette flow are shown on Figure 2.

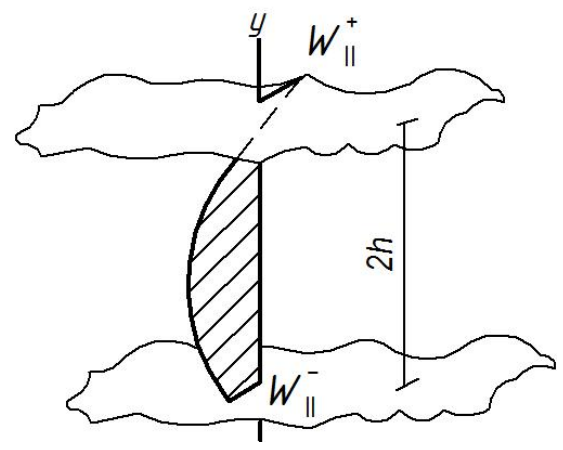

Figure 2. Fragment of the slit channel with boundary conditions

\section{Results and discussion}

We studied the longitudinal flow of non-Newtonian fluid in rectangular channel, which has longitudinal velocities sent on its borders. The distribution of these boundary velocities is displayed on Figure 3.

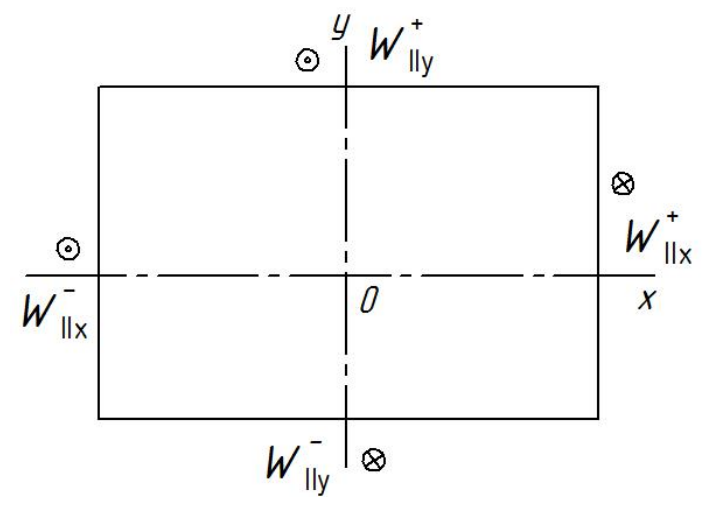

Figure 3. Longitudinal flow in rectangular channel and boundary conditions on its borders

The flow on this figure is a special case of the general three-dimensional flow, shown on Figure 1. Longitudinal flow has one velocity component $v_{2}$, which depends on two coordinates $x$ and $y$ in rectangular cross-section of the channel. The equation of liquid state is defined by viscosity $\mu$, which has the following form:

$$
\dot{I}_{2}=\left(\frac{\partial v_{z}}{\partial x}\right)^{2}+\left(\frac{\partial v_{z}}{\partial y}\right)^{2} \dot{I}_{2}=\left(\frac{\partial v_{z}}{\partial x}\right)^{2}+\left(\frac{\partial v_{z}}{\partial y}\right)^{2}
$$

in which $\alpha$ and $\beta$ - parameters that depend on molecular characteristics of liquid and that are determined by an experiment. The equation of the flow is represented in the following form:

$$
\frac{d P}{d z}=\frac{\partial}{\partial x}\left\{\left(\alpha+\beta \sqrt{\dot{I}_{2}}\right) \frac{\partial v_{z}}{\partial x}\right\}+\frac{\partial}{\partial y}\left\{\left(\alpha+\beta \sqrt{\dot{I}_{2}}\right) \frac{\partial v_{z}}{\partial y}\right\} .
$$


In accordance with the above, this problem should be reduced to a form which corresponds to the problem of longitudinal slit flow. This should be done twice: at first problem (1) should be transformed into problem of slit flow with borders, which are parallel to $o x$ axis; then it should be transformed into problem of slit flow with borders, which are parallel to oy axis. Afterwards, the general flow in rectangular channel is constructed using composition method. Following this plan, it is necessary to express the derivatives $\partial v_{z} / \partial x$ и $\partial v_{z} / \partial y$ through each other. Relationship between the derivatives has the form: $\partial v_{z} / \partial x \sim x \partial v_{z} / \partial y$, where $x=h / a$, while $h$ and $a$ are the sides of the rectangle on the Figure 3. This relationship is defined up to a factor, the form of which depends on the degree of convexity or concavity of the velocity profile. Using derivative $\partial v_{z} / \partial y$ as a primary one, and then derivative $\partial v_{z} / \partial x$ we can come to the following equations of the slit flow:

$$
\begin{aligned}
& \frac{d P}{d z}=\frac{\partial}{\partial y}\left\{\left[\alpha(1+x)+\frac{\beta(1+x)^{\frac{3}{2}}}{2}\left|\frac{\partial v_{z}}{\partial y}\right|\right] \frac{\partial v_{z}}{\partial y}\right\}=0 . \\
& \frac{d P}{d z}=\frac{\partial}{\partial x}\left\{\left[\alpha\left(1+\frac{1}{x}\right)+\frac{\beta\left(1+\frac{1}{x 2}\right)}{2}\left|\frac{\partial v_{z}}{\partial y}\right| \mid \frac{\partial v_{z}}{\partial y}\right\}=0 .\right.
\end{aligned}
$$

Below, both problems are solved simultaneously, since they have the same structure. In order to solve them variables $x$ and $y$ are denoted as $x_{i}$, where $i=x, y$; parameters of both problems, multiplied by factors, which depend on parameter $x$, are denoted as $d_{i}, \beta_{i}$, where $i=x, y$; rectangle sizes $h$ and $a$ are denotes as $l_{i}$, thus $l_{x}=a, l_{x}=h$. By virtue of inclusion of absolute value of velocity derivative, the solution to problem (3) consists of two branches which intersect at point $x_{i}{ }_{i}$

$$
\begin{aligned}
& v_{z^{i}}^{+1}=-\frac{\alpha_{i}}{2 \beta_{i}}+\sqrt{\frac{\alpha_{i}^{2}}{4 \beta_{i}^{2}}+\frac{x_{i}}{\beta_{i}} \frac{d P}{d z}+c_{i}}, \\
& v_{z^{i}}^{-1}=-\frac{\alpha_{i}}{2 \beta_{i}}+\sqrt{\frac{\alpha_{i}^{2}}{4 \beta_{i}^{2}}-\frac{x_{i}}{\beta_{i}} \frac{d P}{d z}+c_{i}} .
\end{aligned}
$$

The choice of characters is dictated by the fact that one branch should have a positive derivative, while the other - a negative one. At point $x_{i}^{*}$ where branches intersect, the derivative turns to zero. This condition along with boundary condition (see Figure 3), lead to the following expressions for velocity $v_{z}^{ \pm}$:

$$
\begin{aligned}
& v_{z^{i}}^{+}\left(x_{i}\right)=\frac{\alpha_{i}}{2 \beta_{i}}\left(l_{i}-x_{i}\right)+\left(\frac{\alpha_{i}^{2}}{4 \beta_{i}^{2}}+\frac{x_{i}-x_{i}^{*}}{\beta_{i}} \frac{d P}{d z}\right)^{\frac{3}{2}} \cdot \frac{2}{3} \frac{\beta_{i}}{\frac{d P}{d z}}-\left(\frac{\alpha_{i}^{2}}{4 \beta_{i}^{2}}+\frac{l_{i}-x_{i}^{*}}{\beta_{i}} \frac{d P}{d z}\right)^{\frac{3}{2}} \cdot \frac{2}{3} \frac{\beta_{i}}{\frac{d P}{d z}}+w_{i}^{+}, \\
& v_{z^{i}}^{-}\left(x_{i}\right)=\frac{\alpha_{i}}{2 \beta_{i}}\left(l_{i}+x_{i}\right)+\left(\frac{\alpha_{i}^{2}}{4 \beta_{i}^{2}}+\frac{x_{i}+x_{i}^{*}}{\beta_{i}} \frac{d P}{d z}\right)^{\frac{3}{2}} \cdot \frac{2}{3} \frac{\beta_{i}}{\frac{d P}{d z}}-\left(\frac{\alpha_{i}^{2}}{4 \beta_{i}^{2}}+\frac{l_{i}+x_{i}^{*}}{\beta_{i}} \frac{d P}{d z}\right)^{\frac{3}{2}} \cdot \frac{2}{3} \frac{\beta_{i}}{\frac{d P}{d z}}+w_{i}^{-} .
\end{aligned}
$$

Values $x_{i}^{*}$, which are calculated from the condition of continuity of velocity $v_{i}$ at points $x_{i}^{*}$, have the following representation $[5,6]$ : 


$$
x_{i}^{*}=-\frac{w_{i}^{+}-w_{i}^{-}}{\frac{l_{i}}{\beta_{i}}-2\left(\frac{\alpha_{i}{ }^{2}}{4 \beta_{i}{ }^{2}}+\frac{l_{i}}{\beta_{i}} \frac{d \mathrm{P}}{d z}\right)^{1 / 2}} .
$$

By integrating expression (5) on interval $\left(-l_{i}, x_{i}^{*}\right)$ for branch $v_{i}$ and on interval $\left(x_{i}^{*}, l_{i}\right)$ for branch $v^{+}{ }_{i}$ we can come to the following expression for the $\dot{v}_{i}$ flow rate:

$$
\begin{gathered}
\dot{v}_{i}=w_{i}^{+}\left(l_{i}-x_{i}^{*}\right)+w_{i}^{-}\left(l_{i}+x_{i}^{*}\right)+\frac{\alpha_{i}}{2 \beta_{i}}\left(l_{i}^{2}+x_{i}^{* 2}\right)+\frac{4}{15}\left(\frac{\beta_{i}}{\left.\frac{d P}{d z}\right)^{2} \times}\right. \\
\times\left\{\left[\left(\frac{\alpha_{i}^{2}}{4 \beta_{i}^{2}}+\frac{l_{i}-x_{i}^{*}}{\beta_{i}} \frac{d P}{d z}\right)^{\frac{5}{2}}-\left(\frac{\alpha_{i}^{2}}{4 \beta_{i}^{2}}\right)^{\frac{5}{2}}\right]+\left[\left(\frac{\alpha_{i}^{2}}{4 \beta_{i}^{2}}+\frac{l_{i}+x_{i}^{*}}{\beta_{i}} \frac{d P}{d z}\right)^{\frac{5}{2}}-\left(\frac{\alpha_{i}^{2}}{4 \beta_{i}^{2}}\right)^{\frac{5}{2}}\right]\right\}- \\
-\frac{2}{3} \frac{\beta_{i}}{\frac{d P}{d z}}\left\{\left(\frac{\alpha_{i}^{2}}{4 \beta_{i}^{2}}+\frac{l_{i}-x_{i}^{*}}{\beta_{i}} \frac{d P}{d z}\right)^{\frac{3}{2}}\left(l_{i}-x_{i}^{*}\right)+\left(\frac{\alpha_{i}^{2}}{4 \beta_{i}^{2}}+\frac{l_{i}+x_{i}^{*}}{\beta_{i}} \frac{d P}{d z}\right)^{\frac{3}{2}}\left(l_{i}+x_{i}^{*}\right)\right\} .
\end{gathered}
$$

The expression (7) can be somewhat simplified by compromising accuracy. To do so, the terms in powers $5 / 2$ and $3 / 2$ should be expanded in Taylor series up to and including the new term by the degree of smallness. The value $\left(x_{i}^{*} d P / d z\right) / \beta_{i}$ should be used as an expansion parameter. This expansion gives an error of not more than $16 \%$ for the case of inequality $\left|x_{i}^{*}\right|<l_{i}$. If such expansion is performed, then expression (7) for the flow rate turns into a square polynomial with respect to the value of $x_{i}{ }_{i}$ The result is as follows:

$$
\begin{aligned}
\dot{v}_{i}=w_{i}^{+}\left(l_{i}-x_{i}^{*}\right)+w_{i}^{-}\left(l_{i}+x_{i}^{*}\right)+\frac{\alpha_{i}}{2 \beta_{i}}\left(l_{i}^{2}+x_{i}^{* 2}\right)+\frac{8}{15}\left(\frac{\beta_{i}}{\frac{d P}{d z}}\right)^{2}\left[\left(\frac{\alpha_{i}^{2}}{4 \beta_{i}^{2}}+\frac{l_{i}}{\beta_{i}} \frac{d P}{d z}\right)^{\frac{5}{2}}-\left(\frac{\alpha_{i}^{2}}{4 \beta_{i}^{2}}\right)^{\frac{5}{2}}\right]-. \\
-\frac{2}{3} \frac{\beta_{i}}{\frac{d P}{d z}}\left[2 l_{i}\left(\frac{\alpha_{i}^{2}}{4 \beta_{i}^{2}}+\frac{l_{i}}{\beta_{i}}+\frac{l_{i}}{\beta_{i}} \frac{d P}{d z}\right)^{\frac{3}{2}}+3 \frac{1}{\beta_{i}} \frac{d P}{d z}\left(\frac{\alpha_{i}^{2}}{4 \beta_{i}^{2}}+\frac{l_{i}}{\beta_{i}} \frac{d P}{d z}\right)^{\frac{1}{2}} x_{i}^{* 2}\right] .
\end{aligned}
$$

With the help of the formulas (7) or (8) it is possible construct a formula for the longitudinal flow rate, but in the rectangular channel, not a slit one. In order to perform such construction, we should use the considerations of limit compliance, which implies that if parameter $x$, which is present in definition of values $\alpha_{i}, \beta_{i}$, tends to zero, we get a slit channel in channel with borders along the $o x$ axis. If, however, this parameter tends to infinity, we get the slit flow in channel with borders along the oy axis. 
Based on these two extreme cases, the rate of the general flow can be represented as the composition of slit flows rate with weighting factors that depend on parameter $x$ and satisfy the compliance with the limit. Such composition is ambiguous. Its final concrete form is determined empirically. However, the simplest kind of composite factors is used. Based on what said above, the form of such composition can be the following:

$$
\dot{v}=\dot{v}_{y}\left(\frac{1}{1+x^{m}}\right)^{n}+\dot{v}_{x}\left(\frac{x^{m}}{1+x^{m}}\right)^{n},
$$

where $m$ and $n$-composition parameters. The simplest choice is to set $n=1$. The following considerations can be made with regards to the value of the parameter $m$. A common feature of slow (Stokes) viscous fluids is that the influence of bounds on the velocity profile shaping distributes over the distance into the flow region of the order of the corresponding border length. From this, it follows that for rectangular channels, that are highly elongated along the $x$ axis, the flow with velocity $v_{y}(y)$ distributes over an area of exponent $a h-h^{2}$, while the flow with velocity $v_{x}(y)$ distributes over the area of exponent $h^{2}$. For channels, that are highly elongated along the oy axis, the situation is exactly the opposite: flow with velocity $v_{x}(y)$ distributes over the area of exponent $a h-a^{2}$, while flow with velocity $v_{y}(y)$ distributes over the area of exponent $a^{2}$. For these the expression for the rates can be represented in the following form:

$$
\dot{v}(x=1)=\dot{v}_{y}(x=0) \frac{a^{2}}{a h}+\dot{v}_{x}(x=\infty) \frac{a h-a^{2}}{a h}
$$

Comparing formulas (9) and (10) we can conclude that (10) leads to the fact that in (9) parameter $n$ should be set to 1 .

Now we must construct the velocity field from the expressions (5). In order to do this we must refer to the Newtonian fluid flow in rectangular channel. Such a flow due to the linearity of the problem can be divided into two: the current, which is caused by the movement of the walls; and the flow caused by pressure drop. For the first flow, the rate is equal to the product of the average speed on the cross-sectional area and the velocity by a factor equal to the quantity ratio of the length of the moving part of the perimeter length of the cross section to the entire perimeter. This fact can also be interpreted in a way that the influence of the moving borders distributes not throughout the entire cross-sectional area but through its part. From physical considerations, it is clear that this part should be adjacent to the moving borders. The remaining part of the cross-section is influence by immovable walls and must adjust to non-moving borders. The influence areas of the borders are qualitatively presented on Figure 4.

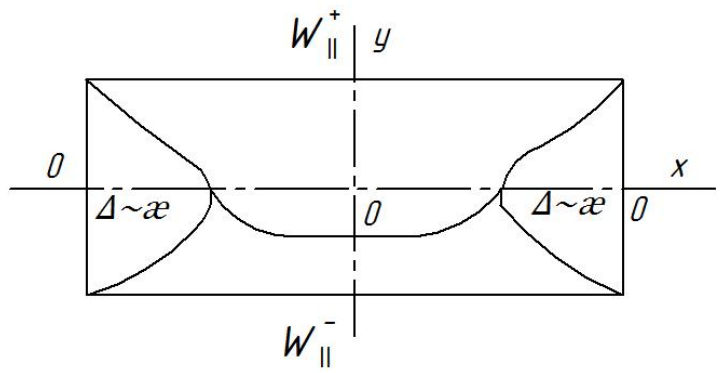

Figure 4. Areas of influences of the boundary conditions on rectangular channel borders: $\Delta$ - the depth of penetration of the zero boundary conditions. 
The figure shows that the size of the area adjacent to immovable borders has the exponent of $x /(1+x)$. From this follows the fact that is all the rectangle area is taken as 1 then the size of the area influenced by borders movement is $1 /(1+x)$. Then for the parts elongated along the cross-sections the remaining area will actually be $x /(1+x)$. From this and from Figure 4 follows the fact that this value is the same as the depth of penetration of influence of zero boundaries. For the second flow slightly different considerations should be used. If the flow rate is taken as 1 and remove a pair of borders which are perpendicular to $o x$ axis, then adding this pair of borders decreases the flow rate to $1 /\left(1+x^{2}\right)$ times. This fact can be interpreted as decreasing the maximum velocity in the same amount of times. The method of composing solutions (5) has the simple nature and can be used in engineering. In lies in using the corresponding solution from (5) for each pair of borders. Areas, in which different solutions work, have the shared borders. The shape of these borders can be defined from conditions of continuity of velocity fields on these borders. If we write continuity conditions for different solutions then there will be four conditions: $v_{z}^{+}(y)=v_{z}^{+}(x) ; v_{z}^{-}(y)=v_{z}^{+}(x) ; v_{z}^{+}(y)=v_{z}^{-}(x) ; v_{z}^{-}(y)=v_{z}^{-}(x)$.

Each of continuity conditions leads to one line. The above statements are presented on Figure 5.

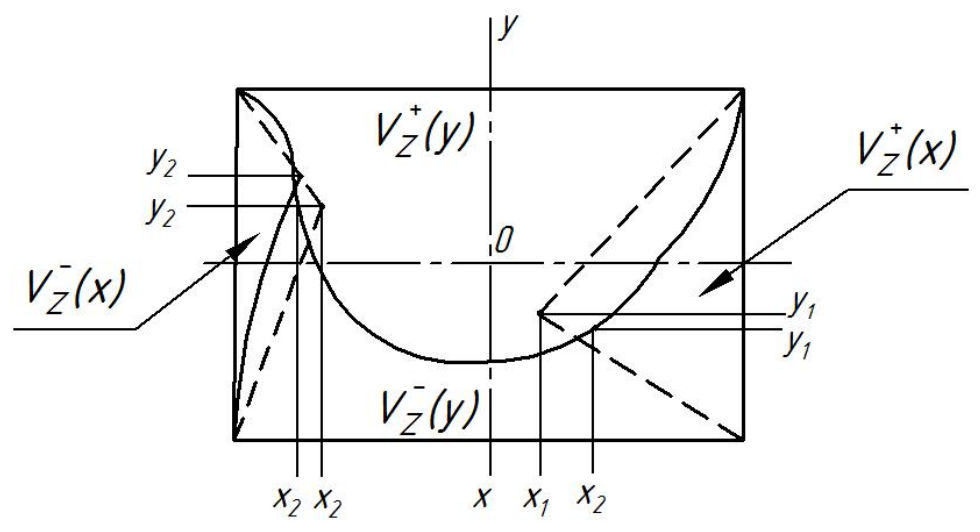

Figure 5. Partitioning of channel cross-section into areas with velocity profiles $v_{z}^{ \pm}(y), v_{z}^{ \pm}(x)$ : solid line - actual partitioning; dashed line - approximate partitioning

The conditions can be represented in the following expanded form:

$$
\begin{aligned}
& a^{-}+b^{-} y+\left(c^{-}+d^{-} y\right)^{\frac{3}{2}}=m^{+}+n^{+} x+\left(l^{+}+p^{+} x\right)^{\frac{3}{2}}, \\
& a^{+}+b^{+} y+\left(c^{+}+d^{+} y\right)^{\frac{3}{2}}=m^{-}+n^{-} x+\left(l^{-}+p^{-} x\right)^{\frac{3}{2}}, \\
& a^{-}+b^{-} y+\left(c^{-}+d^{-} y\right)^{\frac{3}{2}}=m^{-}+n^{-} x+\left(l^{-}+p^{-} x\right)^{\frac{3}{2}}
\end{aligned}
$$

in which values $a^{ \pm,} b^{ \pm,} c^{ \pm,} d^{ \pm,} m^{ \pm,} l^{ \pm,} p^{ \pm}$are defined in the obvious way from (5) and are not shown because of triviality and in order to save space. Equations Equations (11) can be solved (in order to define the form of relationships $\left.y_{i}(x), y=1,2,3,4\right)$ only numerically. Approximate solutions can be obtained by partially linearizing equation (11). To do this we need to make the following notations: 


$$
\begin{aligned}
& \frac{1}{h+y_{2}} \int_{-h}^{y_{2}}\left(c^{-}+d^{-} y\right)^{\frac{1}{2}} d y=\lambda^{-} ; \\
& \frac{1}{a-x_{1}} \int_{x_{1}}^{a}\left(l^{+}+p^{+} x\right)^{\frac{1}{2}} d x=\delta^{+} ; \\
& \frac{1}{a+x_{2}} \int_{-a}^{x_{2}}\left(l^{-}+p^{-} x\right)^{\frac{1}{2}} d x=\delta^{-},
\end{aligned}
$$

in which $y_{1}, y_{2}, x_{1}, x_{2}$ - points, presented on Figure 5. In these notations after linearization, the linear equations are obtained for boundary lines, while actual lines become line segments. Equations for boundary lines acquire the following form:

$$
\begin{aligned}
& \left(a^{-}+c^{-} \lambda^{-}\right)+\left(b^{-}+d^{-} \lambda^{-}\right) y=\left(m^{+}+l^{+} \delta^{+}\right)+\left(n^{+}+p^{+} \delta^{+}\right) x \\
& \left(a^{+}+c^{+} \lambda^{+}\right)+\left(b^{+}+d^{+} \lambda^{+}\right) y=\left(m^{-}+l^{-} \delta^{-}\right)+\left(n^{-}+p^{-} \delta^{-}\right) x \\
& \left(a^{-}+c^{-} \lambda^{-}\right)+\left(b^{-}+d^{-} \lambda^{-}\right) y=\left(m^{-}+l^{-} \delta^{-}\right)+\left(n^{-}+p^{-} \delta^{-}\right) x .
\end{aligned}
$$

Solutions (13) are represented on Figure 5 as contour lines. In order to obtain values $x_{1}$ and $x_{2}$ (see Figure 5) the solutions of first two and the last two equations (13) should be equated in pairs. As a result, for $x_{1}$ and $x_{2}$ we get the following formulas:

$$
x_{2}=\frac{\frac{\left(m^{-}+l^{-} \delta^{-}\right)-\left(a^{+}+c^{+} \lambda^{+}\right)}{b^{+}+d^{+} \lambda^{+}}-\frac{\left(m^{-}+l^{-} \delta^{-}\right)-\left(a^{-}+c^{-} \lambda^{-}\right)}{b^{-}+d^{-} \lambda^{-}}}{\frac{n^{-}+p^{-} \delta^{-}}{b^{-}+d^{-} \lambda^{-}}-\frac{n^{-}+p^{-} \delta^{-}}{b^{+}+d^{+} \lambda^{+}}}
$$

Since $\lambda^{ \pm}$and $\delta^{ \pm}$are unknown, they should be determined by the equations (12) and (13), where values $y_{1}$ и $y_{2}$ should be defined as well. The system of equations for finding $\lambda^{ \pm}$ and $\delta^{ \pm}$is nonlinear and is solved numerically.

For velocity profiles $v_{z}^{i}$ for which $y^{*}$ and $x^{*}$ lie correspondingly in intervals $(-h, h)$ and $(-a, a)$, the non-numeric solution can be obtained. The expressions for $v_{z}{ }^{i}$ near the points $y=$ $\pm h$ and $x= \pm a$ should be expanded in Taylor series up to and including the first term. The results of these expansions can be written in the following form:

$$
\begin{aligned}
& v_{z}^{-}(y)=w_{y}^{-}+\left[\frac{\alpha_{y}}{2 \beta_{y}}+\left(\frac{\alpha_{y}^{2}}{4 \beta_{y}^{2}}+\frac{h-y^{*}}{\beta_{y}} \frac{d P}{d z}\right)^{1 / 2}\right](h+y), \\
& v_{z}^{+}(x)=w_{x}^{+}+\left[\frac{\alpha_{x}}{2 \beta_{x}}-\left(\frac{\alpha_{x}^{2}}{4 \beta_{x}^{2}}+\frac{a-x^{*}}{\beta_{x}} \frac{d P}{d z}\right)^{1 / 2}\right](a-x), \\
& v_{z}^{-}(x)=w_{x}^{-}+\left[\frac{\alpha_{x}}{2 \beta_{x}}+\left(\frac{\alpha_{x}^{2}}{4 \beta_{x}^{2}}+\frac{a+x^{*}}{\beta_{x}} \frac{d P}{d z}\right)^{1 / 2}\right](a+x) .
\end{aligned}
$$

In order to save space we denote multipliers before $h \pm y$ and $a \pm x$ as $M, N, R, S$ correspondingly. The equations of boundary lines will then be written in a following way: 


$$
\begin{aligned}
& y=h-\frac{\left(w_{x}^{+}-w_{y}^{+}\right)+R \cdot a}{M}-\frac{R}{M} x ; \\
& y=-h-\frac{\left(w_{x}^{+}-w_{y}^{-}\right)+R \cdot a}{N}-\frac{R}{N} x ; \\
& y=-h+\frac{w_{x}^{-}-w_{y}^{-}+S \cdot a}{N}+\frac{S}{N} x .
\end{aligned}
$$

Values for coordinates $y_{1}, x_{1}$ и $y_{2}, x_{2}$ are obtained from equations (16) from the following relations:

$$
\begin{gathered}
2 h-\frac{w_{x}^{+}-w_{y}^{+}+S \cdot a}{M}-\frac{w_{x}^{-}-w_{y}^{-}+S \cdot a}{N}=\left(\frac{1}{M}+\frac{1}{N}\right) S \cdot x_{2}, \\
y_{1}=h-\frac{w_{x}^{+}-w_{y}^{+}+R \cdot a}{M}-\frac{R}{M} x_{1}, \\
y_{2}=h-\frac{w_{x}^{+}-w_{y}^{+}+S \cdot a}{M}+\frac{S}{M} x_{2} .
\end{gathered}
$$

\section{Conclusion}

The results that we received, allow us to construct the field of longitudinal flow in rectangular channel with moving borders. At the base of the construction is the solution of one-dimensional problem of slot flow. The composition of flows in slot channels with mutually perpendicular pairs of borders allows to receive the flow rate formula, which satisfies the principle of limit correspondence between flows in rectangular and slot channels [10]. We proposed the method of constructing the velocity field, which in essence, is the partition of the final cross-section of the channel into sections with different dependency on coordinates in a way that on some sections velocity depends only on one coordinate while on others - only on another coordinate. We established the equations for lines, which divide these regions. We suggested methods to define shapes of divider lines: one of them is the rectifications of these lines. For this method, the coordinates of divider lines intersection are fully defined by explicit formulas.

Analyzing formulas (5), which are received from equations (3) it should be noted that the reduction of the original problem with velocity dependency on two coordinates to two problems, in each of which velocity depends on only one coordinate, was based on assessment of relation of derivatives $\partial v_{z} / \partial y \kappa{ } \partial v_{z} / \partial x$. Authors chose the simplest assessment. Objectively, this assessment is valid in the case when differences of velocities $w_{y}^{+}-w_{y}{ }^{-}$ and $w_{x}^{+}-w_{x}{ }^{-}$do not differ very much one from another. The more accurate assessment leads to the following relation between derivatives:

$$
\frac{\partial v_{z} / \partial y}{\partial v_{z} / \partial x} \approx x \frac{w_{y}^{+}-w_{y}^{-}}{w_{x}^{+}-w_{x}^{-}} .
$$

This assessment is valid for such flows, velocity profiles of which are not too convex of bent. These flows are correspondent by values $y^{*}$ and $x^{*}$, which are outside the intervals $(-h, h)$ and $(-a, a)$ correspondingly. Formally, in this case, the formulas do not change their 
appearance. For strongly convex or concave velocity profiles when the contributions of pressure exceeds the contribution of moving channel walls, the assessment of relation of derivatives can be written in a following way:

$$
\begin{array}{ll}
\frac{\partial v_{z} / \partial y}{\partial v_{z} / \partial x} \approx x \frac{c_{1}\left(w_{y}^{+}-w_{y}^{-}\right)+c_{2} v_{m y} l\left(y^{*}\right)}{c_{3}\left(w_{x}^{+}-w_{x}^{-}\right)+c_{4} v_{m x} l\left(x^{*}\right)}, & v_{m y} \equiv \max v_{z}(y), \\
v_{m x} \equiv \max v_{z}(x),
\end{array}
$$

where $c_{1}, c_{2}, c_{3}, c_{4}$ are constants, which depend on flow characteristics.

In conclusion, it must be emphasized that the general form of the formulas in the present work does not depend on the choice of assessment of the relation of derivatives, because these assessments are included into values $\alpha_{y}, \beta_{y}, \alpha_{x}, \beta_{x}$ as factors.

\section{References}

1. Ulev L.M. (2006), Laminarnyie techeniya $v$ soosnyih konicheskih kanalah, NTU «HPI», Kharkiv.

2. Cantelli A. (2009), Uniform Flow of Modified Bingham Fluids in Narrow Cross Sections, Journal of Hydraulic Engineering, 135(8), pp. 640-50.

3. Chhabra R.P, Richardson J.F. (2008), Non-Newtonian Flow and Applied Rheology, 2nd ed., Oxford, Butterworth-Heinemann.

4. Biletsky E., Petrenko E., Semeniuk D. (2014), Theoretical aspects of non-Newtonian fluids flow simulation in food technologies, Ukrainian food journal, 3(2), pp. 270-79.

5. Haldenwang R., Slatter P.T., Chhabra R.P. (2002), Laminar and transitional flow in open channels for non-Newtonian fluids, Hydrotransport 15, 15th International Conference on the Hydraulic Transport of Solids in Pipes, Banff, Canada, pp. 755-68.

6. Biletsky E., Semeniuk D., Petrenko E. (2013), Determination of the dissipation during the flow of non-Newtonian materials in channels with basic geometry, Industrial Technology and Engineering, № 4(9), pp. 5-10.

7. Burdo O.G., Kalinin L.G. (2008), Prikladnoe modelirovanie protsessov perenosa tehnologicheskih sistemah, Druk, Odesa.

8. Harlamov S. (2013), Gidrodinamika i teploobmen: Novyie tendentsii i perspektivyi modelirovaniya vnutrennih techeniy, LAP LAMBERT Academic Publishing.

9. Faber T.E. (2001), Fluid dynamics for physicists, Cambridge University Press, Cambridge.

10. Tovazhnyanskiy L.L., Blletskiy E.V., Tolchinskiy Yu.A. (2013), Modelyuvannya techIy nenyutonivskih ridin u kanalah bazovoyi geometriyi, NTU «HPI», Kharkiv. 